Cost-Effective Sampling of Groundwater Monitoring Wells: A Data Review \& Well Frequency Evaluation

M. Ridley, D. MacQueen

February 16, 2005

American Society of Civil Engineers Anchorage, AK, United States

May 15, 2005 through May 19, 2005 
This document was prepared as an account of work sponsored by an agency of the United States Government. Neither the United States Government nor the University of California nor any of their employees, makes any warranty, express or implied, or assumes any legal liability or responsibility for the accuracy, completeness, or usefulness of any information, apparatus, product, or process disclosed, or represents that its use would not infringe privately owned rights. Reference herein to any specific commercial product, process, or service by trade name, trademark, manufacturer, or otherwise, does not necessarily constitute or imply its endorsement, recommendation, or favoring by the United States Government or the University of California. The views and opinions of authors expressed herein do not necessarily state or reflect those of the United States Government or the University of California, and shall not be used for advertising or product endorsement purposes. 


\title{
COST-EFFECTIVE SAMPLING of GROUNDWATER MONITORING WELLS: A Data Review \& Well Frequency Evaluation
}

\author{
Maureen Ridley ${ }^{1}$ and Don MacQueen ${ }^{2}$ \\ ${ }^{1}$ Lawrence Livermore National Laboratory, P.O. Box 808, L-528, Livermore, CA 94551-0808; \\ PH: (925) 422-3593; FAX (925) 422-2095; Email: ridley1@1lnl.gov \\ ${ }^{2}$ Lawrence Livermore National Laboratory, P.O. Box 808, L-629, Livermore, CA 94551-0808; \\ PH: (925) 423-1062; FAX (925) 422-8684; Email: macq@1lnl.gov
}

\begin{abstract}
Lawrence Livermore National Laboratory (LLNL) uses the Cost-Effective Sampling (CES) program for reviewing groundwater data and optimizing the site's groundwater monitoring plan. The CES program produces a data assessment sheet and a lowest-frequency sampling schedule for each groundwater monitoring location. The assessment sheet and recommended sampling schedule greatly streamline the data review process and provide useful information for regulatory and remedial decision-making. The determination of sampling frequency for a given location is based on trend, variability, and magnitude statistics. The underlying principle is that a location's schedule should be determined primarily by the rate of change in concentrations observed there in the recent past. The larger the rate of change, whether upward or downward, the greater the need for frequent sampling. Conversely, where little change is observed, less sampling is recommended. In 1992, CES was approved by the U.S. EPA - Region IX and the local regulators for use at LLNL, and became part of the LLNL's approved compliance monitoring plan (Lamarre et al. 1996). Applying the CES methodology produced, initially, a 40\% reduction in the annual number of required groundwater samples, and with recent optimization of the program a 55\% reduction has been produced. This reduction saves LLNL \$530,000 annually in sampling, data management, and analysis costs.
\end{abstract}

\section{Introduction}

The initial development of the CES program at Lawrence Livermore National Laboratory (LLNL) was motivated by an overwhelming number of sampling results with little or no change in concentration over many years. This suggested that the monitoring wells were being sampled more often than necessary. CES was developed not only to make sampling frequency recommendations, but also as a data review tool. It provides the project staff with a summary status report of all the chemicals of concern (COC.) The status report includes past sampling frequencies for each location, the recommended sampling frequencies for each COC, the last sample date, the total number of samples, the number of non-detects, and the overall recommendation at the each location. The project staff may increase sampling frequencies above the recommendations of the CES program, for example to adjust for activities such as a new extraction wells coming online, or to track a plume edge.

\section{Locational vs. Data-Oriented Sampling Rationales}


The original method for determining sampling frequencies at LLNL used the well location with respect to the contaminant plume (within $1000 \mathrm{ft}$ of a plume or within a plume) to determine the sampling frequency. Unfortunately, the plumes at LLNL covered a large part of the site and most of the wells were within a plume or within $1000 \mathrm{ft}$ of a plume. The major problem with this method was that it did not take into account the very slow groundwater flow and a contaminant migration of only a few feet per year at the site. In reviewing the groundwater data, it became obvious that the concentrations in many wells tended to remain constant over a long period of time.

This concentration consistency brought about the idea of basing the sampling frequency on the changes in concentration at a given well, rather than the well's location with respect to the plume. CES calculates quantitative measures of the trend and variability of important COCs at each monitoring location. It then interprets this information by means of decision trees to arrive at a recommended sampling frequency. An essential aspect of the CES program has been to use simple statistics within a decision-logic framework to provide information that can be easily understood by any environmental professional.

The question may arise as to why LLNL developed this program instead of using something that was already available. Unfortunately, in 1992 only a limited amount of statistical guidance was available for sites wishing to reduce their sampling schedules in a non-arbitrary manner. EPA documents written for RCRA facilities (U.S. EPA, 1992) suggest using techniques such as Darcy's Equation to estimate the time between independent samples of groundwater based on the physics of flow. Another EPA publication presents a method for estimating sampling intervals from a combination of a first-order autoregressive model of groundwater time series data and the standard error of that series (Barcelona et al., 1989). A third, and especially interesting approach is the creation of temporal variograms to estimate time correlations among samples in the same way that spatial variograms are used to estimate spatial correlations (Tuckfield, 1994). All the above approaches are geared toward determining the time-interval at which statistical independence is achieved. This is a key assumption to the proper application of standard significance tests and also provides a logical foundation on which to base sampling frequencies. However, these more purely statistical approaches to the sampling frequency problem have more difficulty gaining acceptance because of the highly specialized knowledge required to properly implement them. CES is a simple model that requires no specialized statistical knowledge. CES requires only a basic understanding of analytical data.

The data elements necessary to run the CES program are of two types (1) analytic information used for the calculations (location name, chemical compound name, sample date, detection indicator, detection limit, analytical result, and measurement units) and (2) auxiliary information used to assure data integrity (including but not necessarily limited to location coordinates, hydrologic zone [aquifer designation], analytical method, QA flag, and unique sample identifier). Location coordinates, for example, can be used to insure that each location name has unique location coordinates and that each location has a unique name. The hydrologic zone data is sometimes used to analyze data from each zone separately. If LLNL is analyzing another site's data, the data set is most commonly delivered to LLNL in an ASCII file format.

\section{Decision Logic Charts}


The CES decision logic can be used for any type of sampling, as long sample collection and analysis methods have been fairly consistent over a specific period of time. CES could be used just as easily for treatment facility sampling as for groundwater sampling data, however the sampling frequencies would likely be very different. LLNL started with the traditional default sampling frequencies of annual, semi-annual, and quarterly, and later added a biennial (once every two years) option. The initial decision logic was designed with traditional sampling frequencies (annual, semiannual, and quarterly) in mind.

Each scheduling category (frequency) is associated with a base rate of change. At LLNL the annual category is reserved for trends of less than $10 \mathrm{ppb}$ change per year. The quarterly category is associated with rates of change in excess of $30 \mathrm{ppb}$ change per year. The semiannual category falls in the range of 10 - $30 \mathrm{ppb}$ change per year. High and low degrees of variability can move a particular location out of the semi-annual and into the quarterly or annual category. These ranges were developed for concentrations of less than $250 \mathrm{ppb}$ for eleven primary COCs at LLNL (Carbon tetrachloride, chloroform, 1,1-DCA, 1,2-DCA, 1,1-DCE, 1,2DCE, Freon 113, PCE, 1,1,1-TCA, TCE, and Freon 11).

In 1997, more decision logic was added to the program to address higher concentrations, into the high ppm levels. These use rate-of-change categories designed specifically for the analytes and the concentration ranges of interest. Categories have been developed for HMX and RDX, eight metals (arsenic, barium, chromium, copper, lead, nickel, uranium, and zinc) and tritium. The lower rates of change used for the VOCs listed above are commonly seen at arid and semi-arid sites.

The overall decision logic of CES is shown in Figure 1. For a location (usually a groundwater monitoring well or piezometer) to be eligible for consideration, there must be at least six data points within a 12 to 18 month period of time, with a minimum of 2 months between each data point. The decision rules are applied independently to each contaminant in the target list at every location. The frequency assigned to a location is the most frequent schedule estimated for any individual contaminant at that location.

The evaluation of each contaminant proceeds in three steps. First, an initial estimate of the desirable schedule is obtained by analyzing the trend and variability of recent data ("recent" can be specified by the user). In step 2, the trend of the recent data is compared with the trend of the overall data. In step 3, a correction is made for the less toxic substances on the list. Even though their rates of change may be relatively high, their estimates are revised downward so long as the magnitude of the concentrations involved fall below specific limits, such as a maximum contaminant level (MCL). Finally, all CES recommendations are subject to change as a result of scientific and engineering review. The most common reasons for overriding recommendations are anticipation of future remedial actions and public relations considerations pertaining to offsite locations.

Step 1: As mentioned earlier, the primary focus of CES is on trends or rates of change. This is currently defined as the least-squares slope obtained by regressing measured concentration against time. The advantage of this statistic is its ease of interpretation. The slope represents an average yearly change in concentration. A disadvantage is that its suitability for use with nonnormal data is questionable. Sometimes this can be solved by linearizing the data by means of a $\log$ transformation. However, this introduces interpretation problems - the slope no longer 
represents an average yearly change - which we are trying to avoid. Another potential problem is that concentration histories are not always approximately linear. This is addressed in two ways. First, the recent data is given more weight in the algorithm, and the shorter time period represented by the more recent data is more likely to be approximately linear. Second, when the recent and long-term trends are sufficiently different, CES specifies that human intervention is necessary. Finally, despite the imperfections of linear modeling of concentration time-series, CES has been found to be effective.

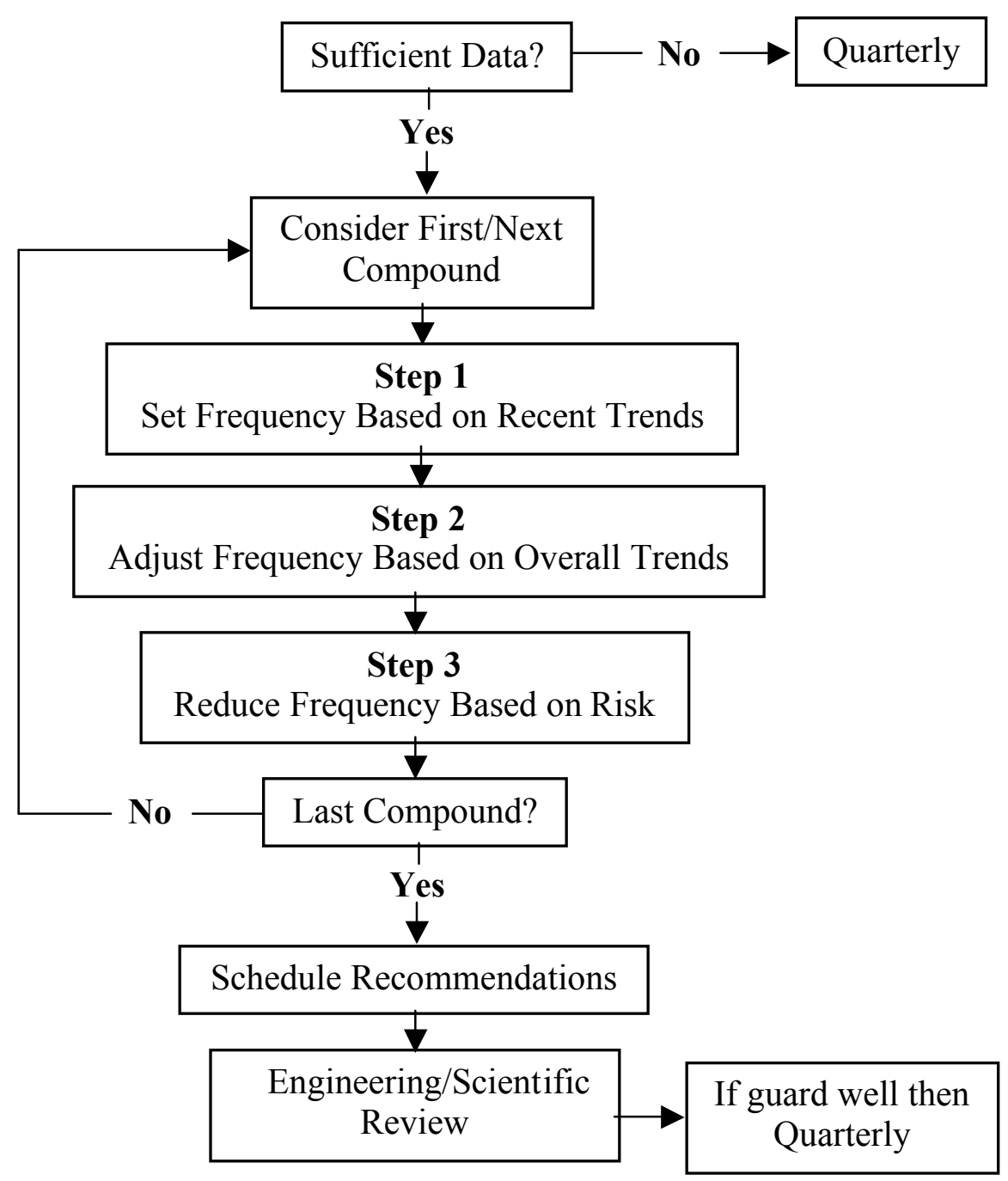

Figure 1. Overview of Steps in CES

Rate, not direction, of change is the dominant factor (see Figure 2). All rate and rate-related statistics use absolute values. Based on the rate of change information, a location is routed along one of four paths (see Figure 3). At LLNL, the lowest rate, 0-10 ppb per year, always leads to an annual frequency schedule. The highest rate, $30+\mathrm{ppb}$ per year, always leads to a quarterly schedule. Rates of change in between 10 and $30 \mathrm{ppb}$ are qualified by variability information, with higher variability leading to a higher sampling frequency. 


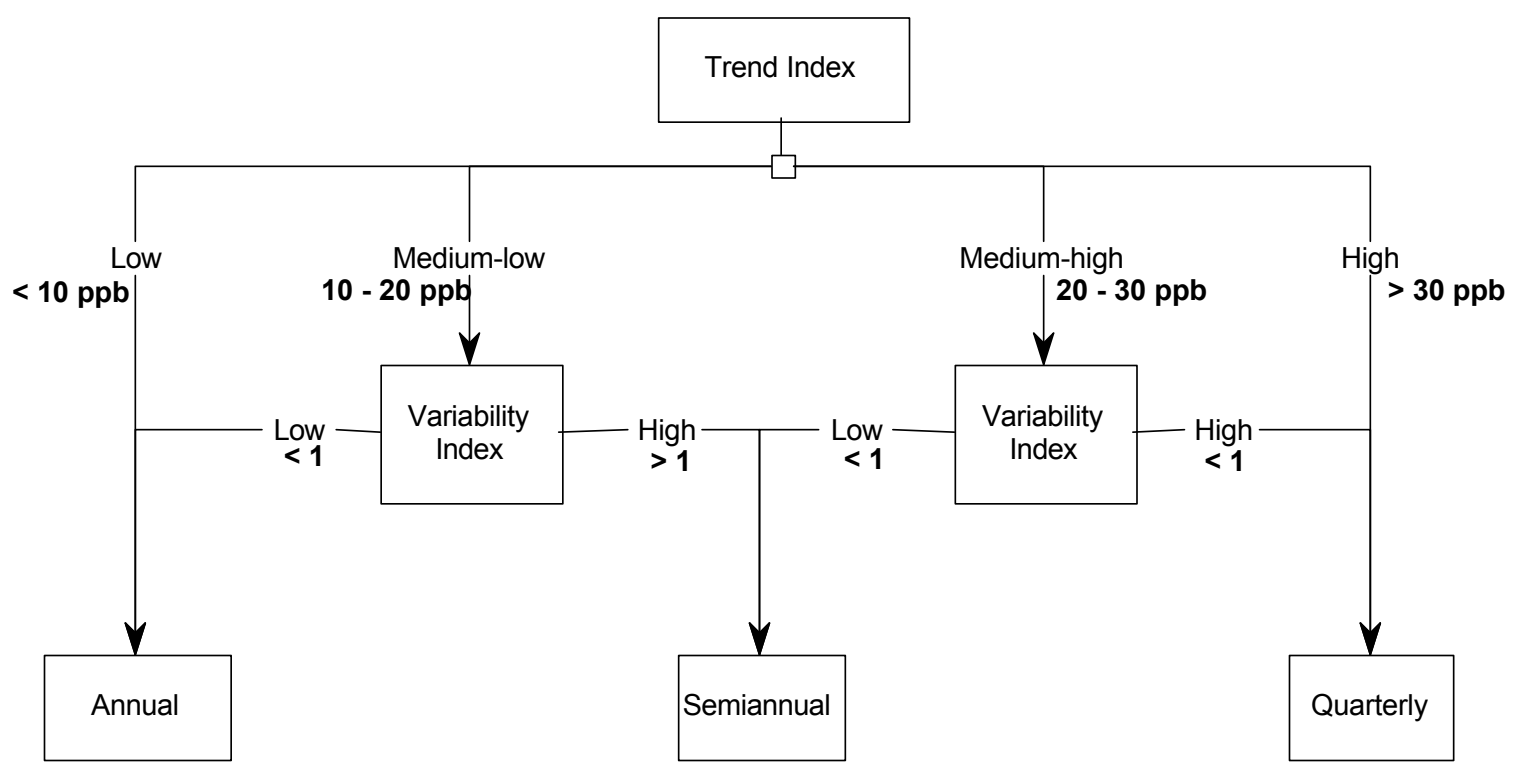

Figure 2. Step 1 : Set Frequency Based on Trend and Variability

Wells with higher concentrations use different sets of parameter ranges. The parameter ranges are determined based on the type of contaminant (VOCs, semi-volatiles, metals, etc.), the groundwater velocity, and the amount of contaminant retardation. If the compound has a decay rate or is being reduced/oxidized, this would also affect the parameter ranges. For some types of compounds such as metals, there need to be specific sets of parameter ranges for each different metal (chromate, arsenic, lead, etc.), because of the differences in subsurface transport processes.

Variability is characterized by a distribution-free version of the coefficient of variation: the range divided by the median concentration. This statistic corrects for the influence of magnitude on variability, which is an important consideration given that the range of concentrations in VOCs routinely varies over three orders of magnitude. The cut-off of 1.0 distinguishing high vs. low variability was derived empirically from the data distributions. It is approximately the median value of that statistic calculated for the two most active contaminants at LLNL, TCE and PCE, across all locations in a benchmark data set.

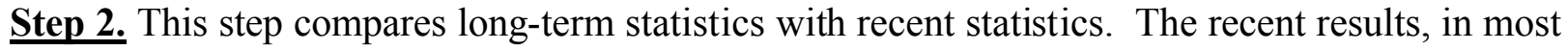
cases, are the dominant decision factors. If there is too large a difference between the recent statistics and the long-term statistics, the program recommends a human examination of the data. Large discrepancies can be the result of a large recent change in the data (increase or decrease), an anomalous data point, or a significant change in the overall data trend. These occur in fewer than a hand full of wells each quarter at LLNL and have been very helpful in detecting outliers, mis-labeled samples, and changing conditions in an area. 


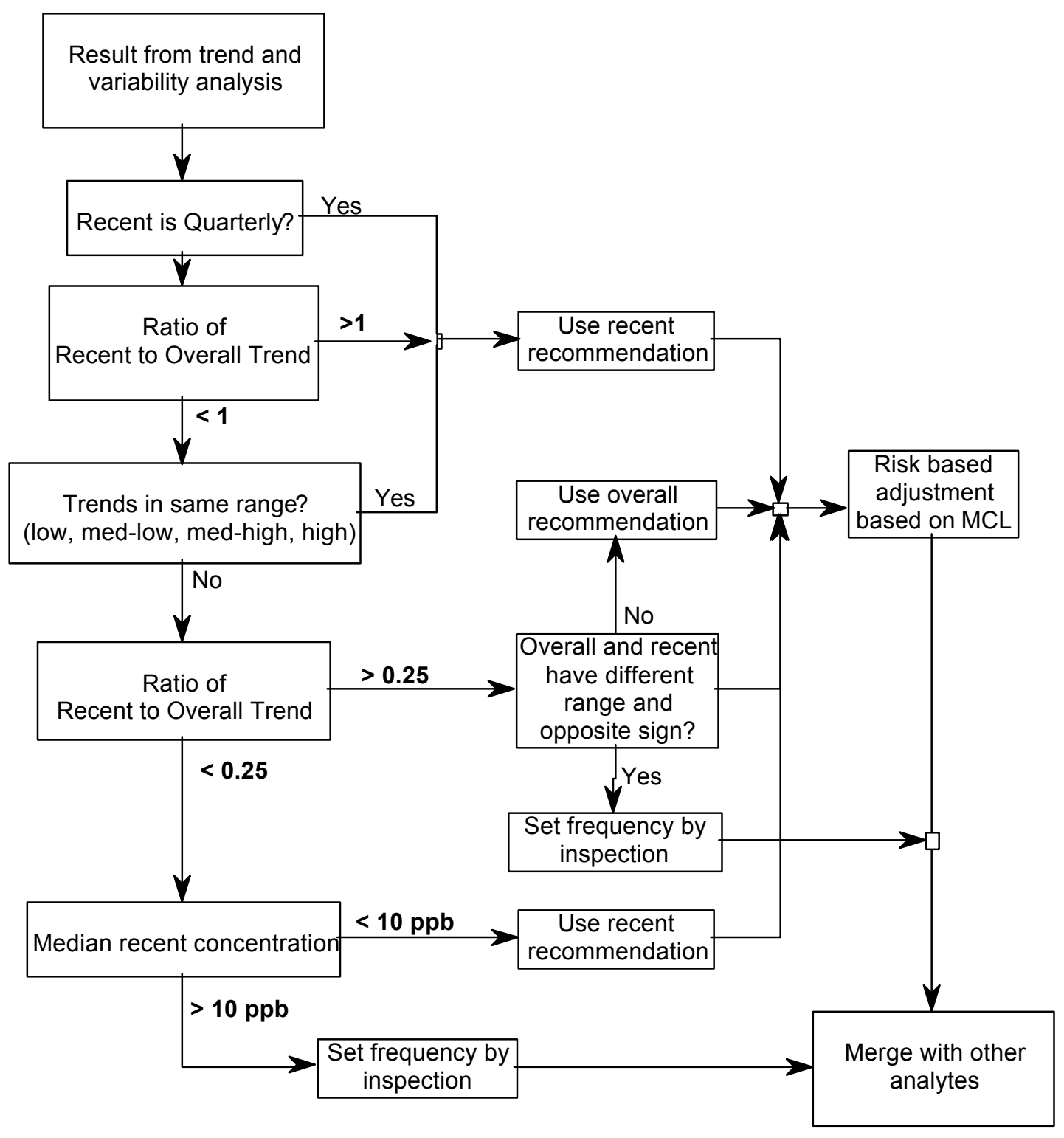

Figure 3. Step 2 of CES - Adjustments Based on Recent Vs. Overall Trends

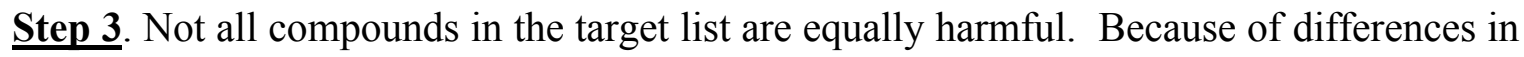
drinking water standards, an average trend of $25 \mathrm{ppb} /$ year for TCE is far more serious than the same trend for Chloroform or the two forms of Freon. So, quarterly and semi-annual decisions are reduced one level if the maximum concentration in the recent set of samples is less than $1 / 2$ of the respective compound's MCL. After these steps have been applied independently to each contaminant in the target list for a particular location, the schedule assigned to the location is the most frequent schedule estimated for any individual contaminant. Thus, it makes sense for all of the chemicals in a target list to be part of the same analytical method (e.g., one of the target lists at LLNL consists of eleven VOCs all of which are included in the EPA 601 method (40 CFR Part 136, Appendix A). 


\section{Application of CES}

The initial application of CES includes a full review of the data set, to make sure the data is in usable condition. This includes checking for data outliers, for consistent use of the same name for each distinct location, for a sufficient number of samples (the minimum six data points) and for other data gaps. Data outliers are checked to see if the outlier can be explained, such as by a mislabeling of the sample or a field problem. If an outlier has no explanation, then the data remains within the data set. One outlier in a large data set should have little effect unless it is a very recent point. As a general rule, no data is removed from a data set unless there is strong evidence of laboratory, handling, or field problems or mistakes.

After the data has been reviewed and cleaned up, CES parameters are developed. There are no hard and fast rules for setting up the parameters. The main goal is to come up with a set of values that once applied, make sense to the technical staff. One of the main objects of CES is to create a program that produces the same results as if a person had reviewed all of the data by hand and made recommendations. After this has been accomplished CES becomes a tool that can be used with confidence in the future. The bottom line is: do the recommendations make sense? If not, the parameters need to be adjusted.

Application of CES at LLNL. A few weeks before the each new quarter begins, the CES program is run. In order to have the most up to date data possible, fast data processing and easy reliable access to the data are essential. At LLNL, with about 400 wells submitted to CES each quarter, the CES calculations and chemical review take less than one day, and the hydrological review is 2 to 3 days. Review at LLNL includes web-based access to plots of all of the analytes at all of the wells.

Application of CES at other sites. The CES program, like any other model, is only as good as the data that goes into the program. Assessing the quality of a data set for use in a modeling program seems like a fairly straightforward task. However, CES has been applied at many different sites and, in every case the most time consuming step is the data gathering and checking to ensure there is appropriate data quality and consistency. One of the most common misconceptions is that if the data is in a data management system (DMS), then it is being well managed. Data are only well managed if a DMS has been well designed and maintained. For the initial application of the CES program, the average time a project spends on gathering and checking the data is about $80 \%$ of the total project time.

The initial application of CES includes a full review of the data set to make sure it is in a usable condition for the CES program, and to produce a snapshot of what the data say are the compounds of concern for the site. The review includes:

- Outliers. Outliers are examined to see if a cause or correction can be found (mislabeled samples, laboratory errors, sampling issues, etc.). If no justification can be found for an outlier to be removed, the data point remains in the data set.

- Detection limit consistency. Detection limits can vary greatly, depending on the types of analysis. Older data may have higher detection limits. Depending on the amount of variation, specialized methods may be necessary in order to calculate the regression statistics (Helsel, 2005). Inconsistent detection limits are a common problem with metals data.

- Is there enough data for analysis by the CES program?

- Are there historical gaps in the data set? 
- Does the data demonstrate a pattern, such as seasonal variation?

- What compounds are being consistently detected?

- What compounds are above the maximum contaminant level (MCL), primary remediation goal (PRG) or an action limit (AL). Frequently compounds are above the MCL, PRG or an $\mathrm{AL}$ and the compounds are not being monitored on a routine basis.

- Develop site-specific CES parameters.

After an initial review of the data has been completed, a list of compounds of concern has been developed, and the parameter ranges have been chosen and tested, then the CES program is run. The length of time for the initial data review, parameter range determination, and first CES run depends on the number of well, the number of analytes, and the condition of the data. CES has been setup for several sites now and the average length of time for the entire initial process has been about one full-time person for 30 days. This time can be significantly longer if a site has many different geologically distinct areas, because each area may need to be considered separately. Once the CES program has been setup for a site subsequent quarterly reviews are very fast.

\section{Cost Savings}

The table below presents the sampling status of monitoring wells at LLNL's restoration sites both before, and after the initial application of CES in 1992, and the current status.

Table 1. The Sampling Schedule Before and After the Application of CES for the Ground Water Monitoring Wells at LLNL.

\begin{tabular}{|l|c|c|c|c|}
\hline & Quarterly & Semi-annual & Annual & Biennial \\
\hline Before CES & $\mathbf{4 1 8}$ & $\mathbf{1 1 2}$ & $\mathbf{2 4}$ & - \\
\hline After CES & $\mathbf{2 1 6}$ & $\mathbf{1 2 4}$ & $\mathbf{2 1 4}$ & - \\
\hline Current (2004) & $\mathbf{9 1}$ & $\mathbf{4 2}$ & $\mathbf{5 2}$ & $\mathbf{1 8 9}$ \\
\hline
\end{tabular}

The overall number of monitoring wells at LLNL has decreased due to the conversion of monitoring wells into extraction wells and from the dewatering of wells. Currently, LLNL is exploring adding another sampling frequency (once every three years or once every five years) as the sizes and concentrations of contaminant plumes have been reduced.

\section{Conclusion}

The application of CES at a site not only saves the site time for regular data review; it also typically lowers the amount of sampling by about $55 \%$ and provides a tremendous amount of information and insight about the analytical data. One of the most significant advantages to using the CES program is that a site can address the changing conditions of the site as soon as the data indicates that a change is needed. The site doesn't have to go through a lengthy regulatory review process to obtain approval to make the necessary changes. As stated earlier, CES is not intended to be a black box, but rather a tool which when appropriately applied at a site, will significantly streamline and enhance the data review and sample scheduling processes.

\section{References}


40 CFR Part 136, Appendix A. Methods for organic chemical analysis of municipal and industrial wastewater: Method 601 - Purgeable halocarbons.

Barcelona, M. J., H. A. Wehrman, M. R. Schock, M. E. Sievers, J. R. Karny. Sampling

Frequency for Ground-Water Quality Monitoring. Report \#EPA/600/4-89/032, Washington, D. C., 1989.

Helsel, D.R. Nondetects And Data Analysis - Statistics for Censored Environmental Data, Wiley-Interscience Hoboken, NJ, ISBN 0-471-67173-8, 2005

Lamarre, A.L, E.M. Nichols, L.L. Berg, M.D. Dresen, R.J. Gelinas, R.W. Bainer, and E.N.

Folsom. 1996. Compliance Monitoring Plan for the Lawrence Livermore National Laboratory Livermore Site. A. UCRL-AR-120936.

\section{Acknowledgements}

This work was made possible by support from UC, Lawrence Livermore National Laboratory's Environmental Restoration Division, under the auspices of the U.S. Department of Energy under contract W-7405-ENG-48. Portions were originally published in Ground Water Monitoring and Remediation, Vol. 24, No.1, Winter 2004. 